\title{
Magnetic Properties of Sulfur-doped Graphene
}

\author{
J. Zhu, ${ }^{\text {a, c }}$ H. Park ${ }^{\mathrm{b}}$, R. Podila,,${ }^{\mathrm{a}, \mathrm{c},{ }^{*}}$ A. Wadehra, ${ }^{\mathrm{b}}$ P. Ayala, ${ }^{\mathrm{e}}$ L. Oliveira, ${ }^{\mathrm{a}} \mathrm{J} . \mathrm{He},{ }^{\mathrm{a}}$ A. A. \\ Zakhidov, ${ }^{\mathrm{f}}$ A. Howard, ${ }^{\mathrm{f}}$ J. Wilkins ${ }^{\mathrm{b}}$ and A. M. Rao ${ }^{\mathrm{a}, \mathrm{c}, \mathrm{d}^{*}}$ \\ a. Department of Physics and Astronomy, Clemson University, Clemson, SC, USA \\ b. Department of Physics, The Ohio State University, Columbus, OH, USA \\ c. Clemson Nanomaterial Center, Clemson University, Clemson, SC, USA \\ d. COMSET, Clemson University, Clemson, SC, USA \\ e. Faculty of Physics, University of Vienna, Vienna, AUSTRIA \\ f. Alan G. MacDiarmid NanoTech Institute, The University of Texas at Dallas, \\ Richardson, TX, USA \\ *Corresponding author: rpodila@g.clemson.edu, arao@g.clemson.edu
}

\begin{abstract}
While studying magnetism of $d$ - and $f$-electron systems has been consistently an active research area in physics, chemistry, and biology, there is an increasing interest in the novel magnetism of $p$-electron systems, especially in graphene and graphene-derived nanostructures. Bulk graphite is diamagnetic in nature, however, graphene is known to exhibit either a paramagnetic response or weak ferromagnetic ordering. Although many groups have attributed this magnetism in graphene to defects or unintentional magnetic impurities, there is a lack of compelling evidence to pinpoint its origin. To resolve this issue, we systematically studied the influence of entropically necessary intrinsic defects (e.g., vacancies, edges) and extrinsic dopants (e.g., S-dopants) on the magnetic properties of graphene. We found that the saturation magnetization of graphene decreased upon sulfur doping suggesting that $\mathrm{S}$-dopants demagnetize vacancies and edges. Our density functional theory calculations provide evidence for: i) intrinsic defect demagnetization by the formation of covalent bonds between S-dopant and edges/vacancies concurring with the experimental results, and ii) a net magnetization from only zig-zag edges, suggesting that the possible contradictory results on graphene magnetism in the literature could stem from different defect-types. Interestingly, we observed peculiar local maxima in the temperature dependent magnetizations that suggest the coexistence of different magnetic phases within the same graphene samples.
\end{abstract}

Keywords: graphene, doping, Ferromagnetic ordering, defects, density functional theory, $\mathrm{X}$-ray photoelectron spectroscopy, magnetization 


\section{Introduction}

Carbon nanomaterials are regarded as one of the best-suited platforms for spintronics due to their low density, inherently low spin-orbit coupling, and large spin-flip scattering lengths ${ }^{[1,2]}$. Ideally, any $s p^{2}$ carbon system is expected to exhibit diamagnetic behavior due to the existence of $\pi$-electron orbital magnetism ${ }^{[3]}$. However, the origin of anomalous ferromagnetic ordering and paramagnetic response in $s p^{2}$ carbon systems has puzzled researchers for many decades ${ }^{[4-6]}$. This unexpected presence of magnetic ordering in nanocarbons is a major impediment for realizing long spin-flip scattering lengths required for spintronic applications. Although there have been many efforts to understand the presence of magnetism in pure carbon-based nanomaterials ${ }^{[7-11]}$, many of them have been either controversial or irreproducible. This is likely due to the presence of unintentional magnetic impurities (e.g., residual Fe catalyst particles in carbon nanotubes), poorly characterized defects, and intrinsic topology (e.g., curvature in $\mathrm{C}_{60}$ and nanotubes). Graphene, a two-dimensional atom-thick layer of $s p^{2}$ carbon, is well suited for elucidating the origin of magnetism due to its fairly simple honeycomb lattice with unique electronic and optical properties ${ }^{[12,13]}$. Furthermore, the properties of many carbon nanomaterials (e.g., fullerenes, carbon nanotubes, graphite, and some polycyclic aromatic molecules) are often theoretically derived from their underlying graphene lattice.

Many theoretical studies have predicted that point defects in graphene exhibit a non-zero magnetic moment, which can possibly interact with each other resulting in a long-range ferromagnetic ordering ${ }^{[7,8,14-18]}$. Nair et al. reported a purely paramagnetic behavior in highly defective fluorinated and ion-irradiated graphene, implying the absence of any defect-defect interactions leading to ferromagnetic (FM) ordering ${ }^{[19,20]}$. On the contrary, others have observed signatures of FM in defected graphene indicating possible interactions between defect-induced magnetic moments ${ }^{[21-23]}$. Collectively, both intrinsic defects (e.g., vacancies and edges) and extrinsic dopants (e.g., fluorine dopants, ionirradiation induced pores, and unintentional magnetic impurities) have been proposed to increase paramagnetic response of graphene, and in some cases even cause FM ordering through defect-defect interactions. As we and others have previously shown, the nature of defects plays a critical role in an unexpected magnetic ordering in many nanostructured materials (particularly, nanograined oxides) derived from non-magnetic bulk, for example $\mathrm{ZnO}{ }^{[24-27]}$. Accordingly in this study, we controllably doped graphene nanoplatelets (GnPs) with sulfur (an extrinsic defect) to tune different magnetic interactions between intrinsic (e.g., between vacancies) and extrinsic defects (e.g., vacancy and S-dopant). Our X-ray photoelectron spectroscopy (XPS) studies and density functional theory (DFT) clearly evince the formation of covalent bonds between $\mathrm{S}$ dopants and intrinsic defects. While we observed that pristine GnPs prepared using the chemical exfoliation method exhibited a weak FM ordering due to the presence of intrinsic defects, we found that the 
FM ordering systematically decreased with increasing $S$ dopants suggesting that the interactions between S-dopants and intrinsic defects demagnetize GnPs.

\section{Experimental Procedure}

Grade M GnPs (xGnP-M-5, 99.95 at. \% carbon and 0.05 at. \% sulfur) were purchased from XG Sciences, Inc. (Michigan, USA). Pristine GnPs consist of short stacks of graphene sheets with an average thickness of approximately $6-8 \mathrm{~nm}$ and average size of $5 \mu \mathrm{m}$ (see Fig S1). In the chemical exfoliation process, as-received GnPs (5 g) were exfoliated in 100 $\mathrm{ml}$ of N-Methylpyrrolidone (NMP) for 2 hours using a 1/8" tip sonicator at $120 \mathrm{~W}$, and then vacuum filtered using a $0.45 \mu \mathrm{m}$ nylon membrane. Subsequently, the collected "cake" was re-sonicated using a fresh portion of NMP for 6 hours and vacuum filtered. Finally, the vacuum filtered GnPs were washed in water thrice and oven dried at $130{ }^{\circ} \mathrm{C}$ for 24 hours.

In the sulfur doping experiment, three $100 \mathrm{mg}$ samples of the exfoliated GnP powders were mixed with $99.5 \%$ elemental sulfur, (100 mesh, Alfa Aesar) at nominal concentrations of $1,1.5$ and 3 wt.\% of S using a mortar and pestle (labeled as pristine GnP, 1 wt.\% S GnP, 1.5 wt.\% S GnP and 3 wt.\% S GnP). The mixed GnP-S samples were vacuum-sealed in quartz tubes ( $1 / 4$ " diameter and 6 " in length) and annealed in a furnace at $1000{ }^{\circ} \mathrm{C}$ for 24 hours. After cooling down to room temperature, the quartz tubes containing the samples were gently shaken by hand for homogenizing the sample, and then reheated again at 1000 ${ }^{\circ} \mathrm{C}$ for another 24 hours. Subsequently, the furnace was cooled to $440{ }^{\circ} \mathrm{C}$ for $2 \mathrm{hrs}$ and the sealed quartz tubes were partially pulled out of the furnace to facilitate condensation of any unreacted sulfur at the opposite cold end of the sealed tubes.

The magnetic properties of samples used in this study were measured using a Quantum Design $^{\circledR}$ vibrating sample magnetometer (VSM) and a superconducting quantum interference device (SQUID) magnetometer. The DC temperature dependent magnetic susceptibility was measured both in zero-field-cooled (ZFC) and field-cooled (FC) conditions with temperature ranging from 3 to $150 \mathrm{~K}$. A Dilor XY triple grating monochromator was used for collecting the micro-Raman spectra of all samples with the $514.5 \mathrm{~nm}$ excitation from an $\mathrm{Ar}^{+}$ion laser. XPS studies were performed in a Phi spectrometer equipped with a monochromatic Al $\mathrm{K}_{\alpha}$ source $(1486.6 \mathrm{eV})$, which has an overall resolution of $0.5 \mathrm{eV}$.

The samples were tested for magnetic impurities such as Fe through inductively coupled plasma mass spectrometry (ICP-MS) measurements (X series II, Thermo Scientific) using an internal standard containing $\mathrm{Li}, \mathrm{Y}$, and In with a detection limit of $0.05 \mathrm{ppb}$ (corrected for sample dilution). For the measurements, $10 \mathrm{mg}$ of sample was dissolved in $10 \mathrm{ml}$ of $70 \%$ nitric acid for $24 \mathrm{hrs}$ under constant stirring. Subsequently, $\mathrm{HNO}_{3}$ containing graphene was 
centrifuged, and the supernatant was removed and diluted into $2 \% \mathrm{HNO}_{3}$ for evaluating the presence of Fe impurities.

The calculations are performed using the projected augmented wave formalism ${ }^{[28,29]}$ of DFT as implemented in the Vienna ab initio simulation package ${ }^{[30,31]}$. The exchange correlation potential is approximated by a generalized gradient approximation using the Perdew-Burke-Ernzerhof functional ${ }^{[32]}$. The DFT orbitals are expanded in plane wave basis with a relatively high energy cutoff of $600 \mathrm{eV}$. The vacancies and vacancy clusters are modeled in $10 \times 10 \times 1$ supercells of the two atom unit-cell of graphene (lattice constant: $2.468 \AA$ ), with a $15 \AA$ vacuum in the vertical direction. We used a relatively large supercell to reduce the undesired interaction between vacancies and their images arising from periodic boundary conditions. The reciprocal space is sampled with $5 \times 5 \times 1 k$-point mesh equivalent to the $50 \times 50 \times 1 k$-point mesh for the two-atom unit cell. The same density of $k$-points is used for simulated nano-ribbons with zigzag- and armchair-edges modeled in 160- and 168-atom computational cells, respectively. All the atomic configurations are optimized with spin-polarized DFT.

\section{Results and Discussion}
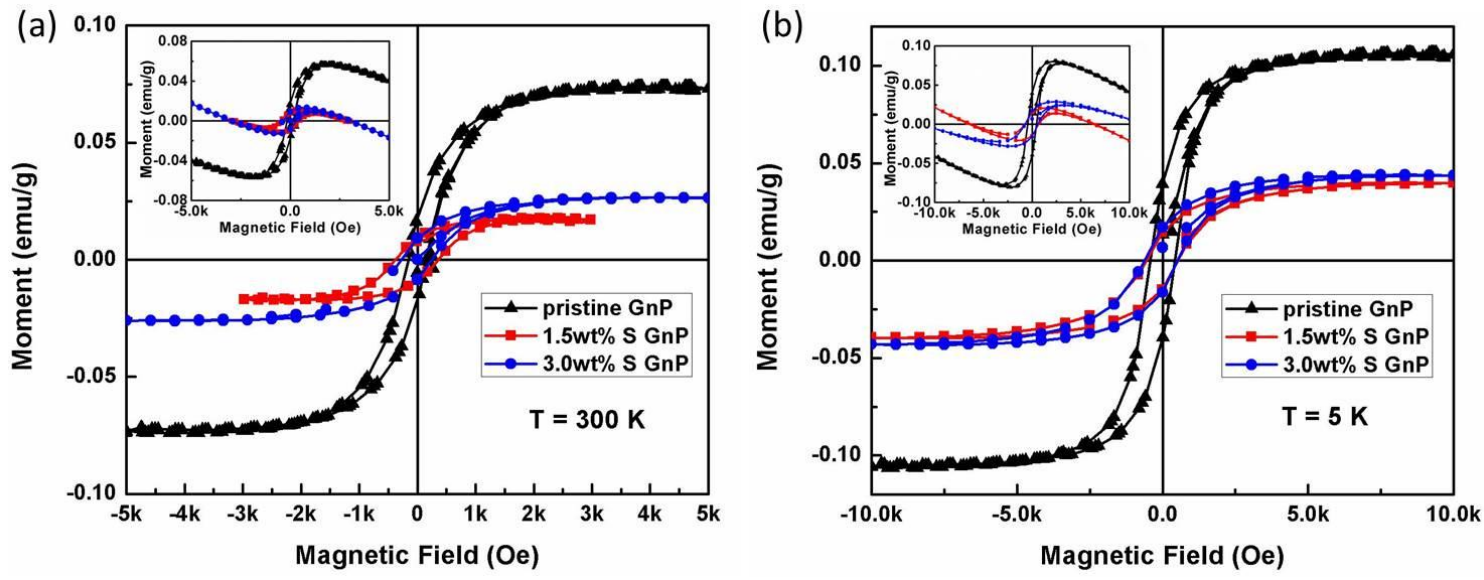

Figure 1. M-H curves for graphitic nanoplatelets, accounted for background diamagnetism, with various $S$ doping concentration measured at (a) $300 \mathrm{~K}$, and (b) $5 \mathrm{~K}$. The insets show the asmeasured M-H data.

As shown in Fig. 1, finite areas of hysteresis loops provide a clear evidence for FM in both pristine and doped $\mathrm{GnP}$ samples at 300 and $5 \mathrm{~K}$, with saturation values $\left(M_{\mathrm{s}}\right) \sim 0.06$ (pristine), 0.017 (1.5 wt.\% S GnP), and $0.043 \mathrm{emu} / \mathrm{g}$ ( $3 \mathrm{wt} . \% \mathrm{~S} \mathrm{GnP}$ ). The FM is embedded in a large diamagnetic (DM) background (see insets in Figs. 1a and b), which arises from the underlying graphene lattice. Although graphite/graphene is diamagnetic, the presence of defects (as it will be discussed later) induce weak FM, similar to nanograined oxides ${ }^{\text {[24-27]. }}$ 
The observed saturation of FM at relatively low fields ( $1.2 \mathrm{kOe}$ at $300 \mathrm{~K})$ for both pristine and doped samples indicates: i) the presence of moderately large spin-clusters, and ii) that the average size of spin-clusters was not affected by doping. The magnetic coercivity $\left(H_{\mathrm{c}}\right)$ increased significantly for all samples at $5 \mathrm{~K}$ (see Table 1) implying that room temperature thermal activation energy $\sim 25 \mathrm{meV}$ is sufficient to induce spin-flip in all $\mathrm{GnP}$ samples. Interestingly, the percent increase in $H_{\mathrm{c}}$ at $5 \mathrm{~K}$ (relative to $H_{c}$ at $300 \mathrm{~K}$ ) is highest for pristine samples $(\sim 250 \%)$ and considerably small in doped samples $(\sim 20 \%)$. Such observations, when juxtaposed with our DFT calculations, could be attributed to dopant-induced passivation of thermal spin fluctuations in unsaturated edge-states of GnPs. Lastly, we observed a non-monotonic decrease of $M_{\mathrm{s}}$ in S-doped samples (see Table 1) confirming that the S-dopants demagnetized GnP samples. Our detailed ICP-MS analysis (Table 2) confirmed that the magnetic response (shown in Fig. 1) is not from magnetic impurities such as Fe, which were found to be $<20 \mathrm{ppb}$ in both pristine and doped samples.

\begin{tabular}{|c|c|c|c|c|c|c|}
\hline & \multicolumn{3}{|c|}{$T=300 \mathrm{~K}$} & \multicolumn{3}{c|}{$T=5 \mathrm{~K}$} \\
\hline & $\begin{array}{c}\text { Pristine } \\
\mathrm{GnP}\end{array}$ & $\begin{array}{c}1.5 \mathrm{wt} \% \mathrm{~S} \\
\mathrm{GnP}\end{array}$ & $\begin{array}{c}3.0 \mathrm{wt} \% \mathrm{~S} \\
\mathrm{GnP}\end{array}$ & $\begin{array}{c}\text { Pristine } \\
\text { GnP }\end{array}$ & $\begin{array}{c}1.5 \mathrm{wt} \% \mathrm{~S} \\
\mathrm{GnP}\end{array}$ & $\begin{array}{c}3.0 \mathrm{wt} \% \mathrm{~S} \\
\text { GnP }\end{array}$ \\
\hline $\begin{array}{c}M_{\mathrm{S}}\left(10^{-2}\right. \\
\mathrm{emu} / \mathrm{g})\end{array}$ & 7.3 & 1.7 & 2.6 & 10.6 & 4.0 & 4.3 \\
\hline$M_{\mathrm{r}}\left(10^{-2} \mathrm{emu} / \mathrm{g}\right)$ & 1.8 & 0.9 & 0.9 & 4.0 & 1.5 & 1.7 \\
\hline$H_{\mathrm{c}}\left(10^{2} \mathrm{Oe}\right)$ & 1.6 & 4.6 & 3.5 & 5.7 & 5.5 & 4.3 \\
\hline
\end{tabular}

Table 1. The value of saturated magnetization $M_{S}$, remnant magnetization $M_{r}$ and coercivity $H_{c}$ for pristine, $1.5 \mathrm{wt} . \%$ and $3.0 \mathrm{wt} . \% \mathrm{~S}$ doped $\mathrm{GnPs}$ under $5 \mathrm{~K}$ and $300 \mathrm{~K}$ obtained from hysteresis loops. The non-monotonic variation of $M_{S}$ could result from sample-to-sample variations, and does not affect our conclusion that $S$-dopants demagnetized GnP samples.

\begin{tabular}{|c|c|c|c|}
\hline & Pristine GnP & 1.5 wt.\%S GnP & 3.0 wt.\%S GnP \\
\hline $\begin{array}{c}\text { Iron concentration } \\
(\mathrm{ppb})\end{array}$ & 17.73 & 9.39 & 12.17 \\
\hline
\end{tabular}


Table 2. ICP-MS analysis of pristine and doped graphene showed only ppb levels of Fe contained in all samples ruling out Fe impurities as a possible cause for the observed magnetic response.
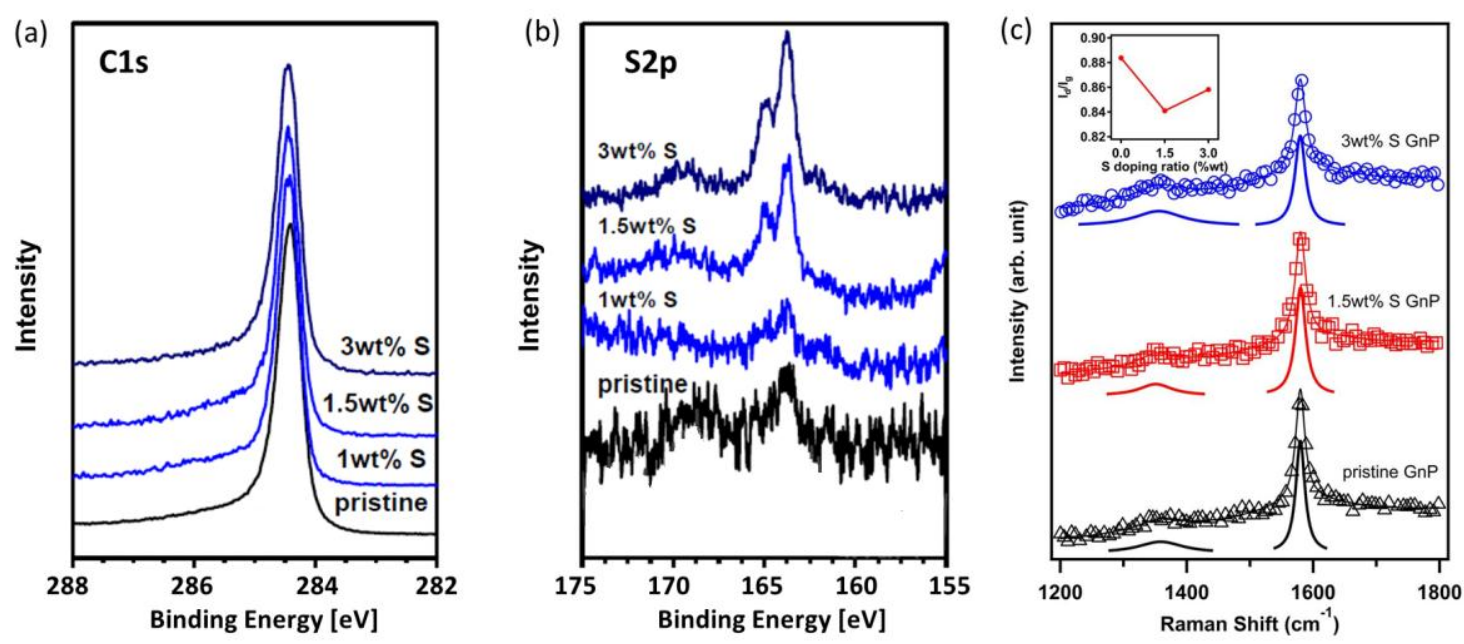

Figure 2. (a) X-ray photoelectron spectra (XPS) recorded for the $C 1$ s line with a photon excitation energy of $1486.6 \mathrm{eV}$ for pristine, $1 \mathrm{wt} . \% \mathrm{~S}, 1.5 \mathrm{wt} . \% \mathrm{~S}$ as well as $3 \mathrm{wt} . \% \mathrm{~S} \mathrm{GnPs}$.(b) XPS recorded for the $S 2 p$ line with a photon excitation energy of $1486.6 \mathrm{eV}$.(c) Micro-Raman spectra of pristine and $S$ doped GnPs. The solid traces below each spectrum represent the deconvoluted fits. The topleft inset shows the $I_{D} / I_{G}$ values for pristine and $S$ doped GnPs.

All samples exhibited an excellent crystallinity as evidenced from C $1 s$ peak in the XPS spectra (Fig. 2a). The S-dopant concentration was quantified from the relative photoemission cross-sections for the $\mathrm{C} 1 s$ and the corresponding $\mathrm{S} 2 p$ peaks. We inferred that pristine, 1 wt. \% S, 1.5 wt. \% S and 3 wt. \% S nominal S-doped GnPs contained 0.05, $0.07,0.18$ and 0.25 at. \% substituted sulfur, respectively. We limited our magnetization studies to pristine, 1.5 , and $3 \mathrm{wt}$ \% samples because the $\mathrm{S}$ concentration in the pristine and 1 wt. \% S samples was not significantly different (see Table S1). Furthermore, our XPS results suggest that $\mathrm{S}$ is substitutionally doped, and the other common forms of S-doping (e.g., $\mathrm{SO}_{2} \mathrm{H}$. $\mathrm{SO}_{3} \mathrm{H}$ ) are not dominant. The Raman spectrum for pristine $\mathrm{GnPs}$ exhibited a weak disorder band (D-band) $\sim 1350 \mathrm{~cm}^{-1}$ in addition to the graphitic band (G-band) $\sim 1585$ $\mathrm{cm}^{-1}$. S-dopants did not significantly alter the intensity of D-band indicating that no additional structural defects (e.g., vacancies or Stone-Wales defects) were created during the doping process (see Fig S3). Collectively, magnetization measurements, XPS and Raman spectra suggest that S-dopants predominantly altered the nature of intrinsic defects without introducing additional structural defects. 

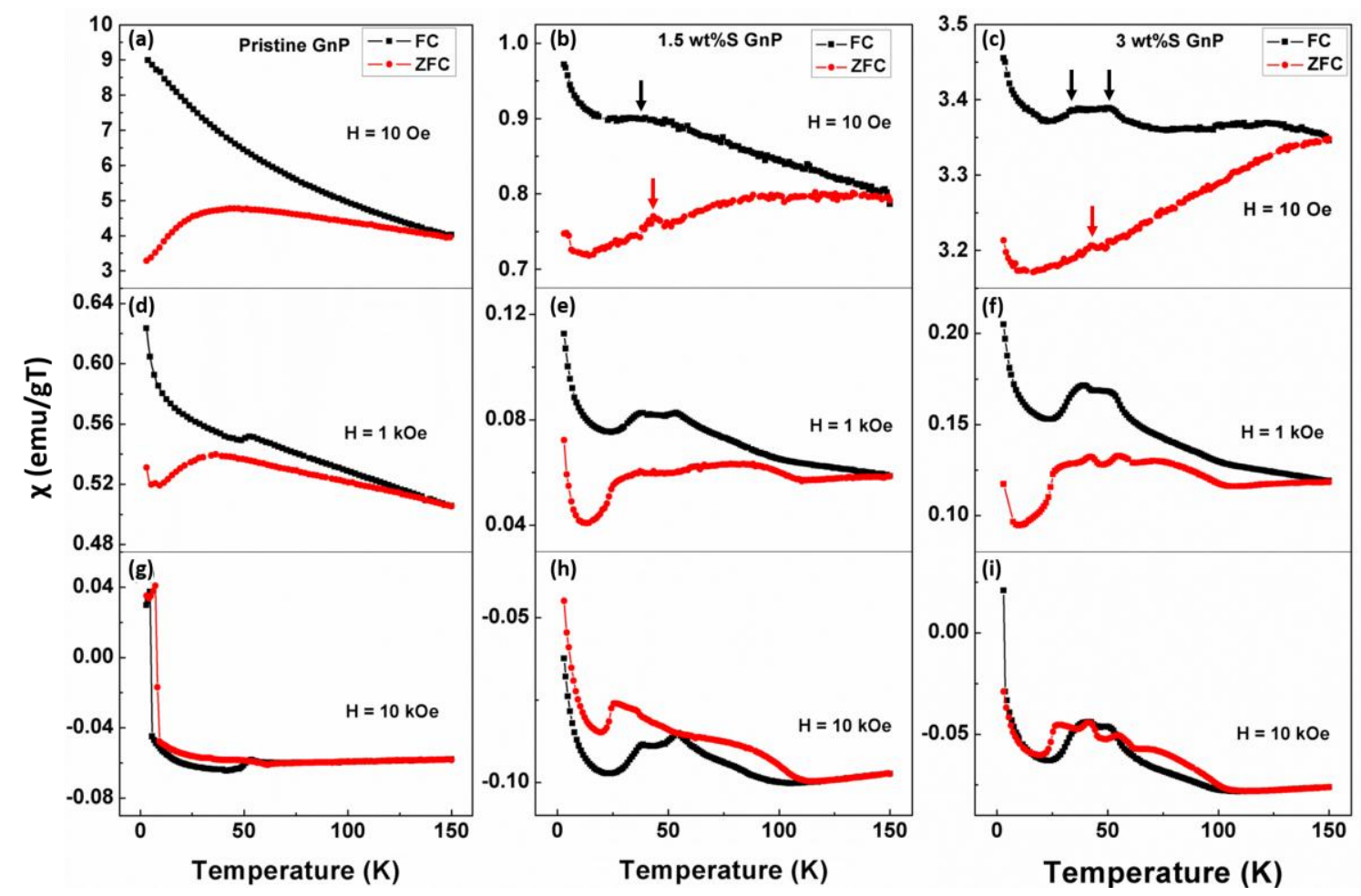

Figure 3. Temperature dependent ZFC-FC data for (a) pristine GnPs, (b) 1.5 wt.\% S GnPs, (c) 3 $w t . \%$ S GnPs in 10 - 10000 Oe. The down pointing arrows are S-dopant induced antiferromagnetic features as discussed in the text.

The following salient features can be gleaned from the zero field cooled and field-cooled (ZFC-FC data; Fig. 3): (i) the low temperature susceptibility $(\chi)$ of the pristine GnPs is about 9 times and 3 times higher than that of 1.5 wt. \% S GnPs and 3 wt. \% S GnPs, respectively, (ii) all samples show very strong temperature dependence in FC cycle $-\chi(\mathrm{T})$ is larger in FC than in ZFC conditions. This is an evidence for the presence of small superparamagnetic clusters in addition to FM domains, (iii) while the FC dependence of pristine GnPs (Fig. 3a) shows a monotonic increase of $\chi(\mathrm{T})$ with decreasing temperature, the ZFC data displays a sharp downturn starting at $~ 38 \mathrm{~K}$, likely due to DM of graphite, (iv) the ZFC data for S doped GnPs exhibit a Curie-like upturn instead of the DM downturn in pristine GnPs at $\sim 38 \mathrm{~K}$, implying that S-dopants have significantly changed the magnetic interactions in GnPs, $(v)$ two new features at $\sim 45$ and $55 \mathrm{~K}$, indicated by arrows in Figs. $3 \mathrm{~b}$ and c, which appear upon $\mathrm{S}$ doping, are possible signatures for antiferromagnetic domains (Fig. S2) ${ }^{[23,33]}$. In fact, we observed that the related intensity of the local maxima increased with increasing magnetic field (Fig 3d-i), confirming such a hypothesis, and (vi) lastly, the splitting between ZFC-FC curves decreased at $10 \mathrm{kOe}$ is consistent with the presence of superparamagnetic clusters in our samples. Interestingly, we observed that the ZFC curve is slightly above the FC (Fig. 3 g-i), which may be attributed to magnetostriction ${ }^{[42]}$. As 
evident from Fig. 3, the temperature dependence of $\chi$ in S-doped graphene presents several rich and intriguing features, which will be discussed in a separate publication. Nonetheless, in summary, our experimental data suggests that more than one type of magnetic behavior can arise from doping and defects in graphene. Indeed, it is evident $\mathrm{GnP}$ samples exhibit a combination of superparamagnetic, ferromagnetic, anti-ferromagnetic (all arising from defects), and diamagnetic (from graphite) domains making GnP a complex magnetic system to be analyzed. To further elucidate the origin and dopant-induced changes in the magnetic properties of GnPs, we performed detailed spin-polarized DFT calculations.

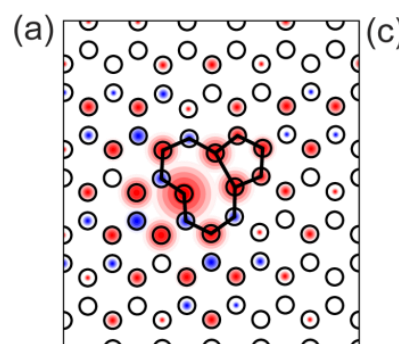

(b)

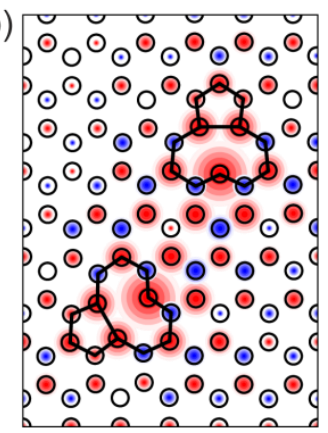

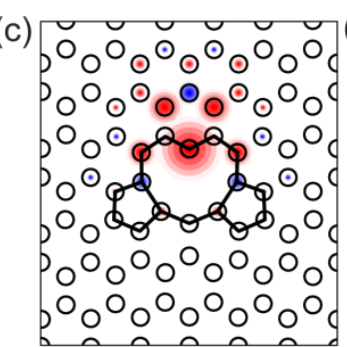

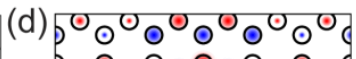

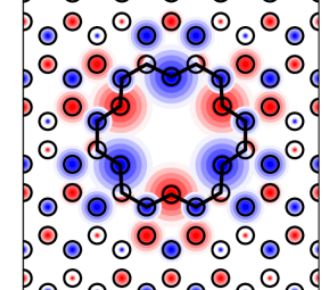

(e)

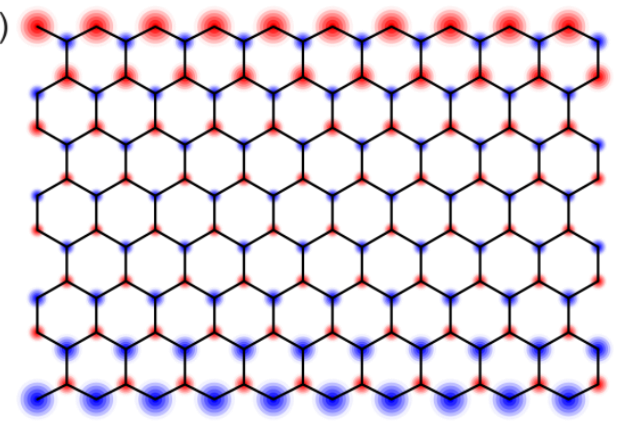

Figure 4. Magnetic structures in graphene and their local spin density plots: (a) monovacancy, (b) two ferromagnetically interacting mono-vacancies, (c) cluster of three vacancies, (d) clusters of six vacancies, and (e) zigzag edges. Up and down local spin densities are represented by circles with red and blue shades, respectively. The magnitude of local moment is represented proportionally to $\log _{10}$ (radius). The net magnetic moment of each structure is (a) 1.38, (b) 2.91, (c) 0.99, and $0.00 \mu_{B}$ for (d) and (e).

Figs. 4a-e represent magnetic structures in defected pristine graphene with multiple vacancy configurations. Upon relaxation, the three dangling-bond sites of the monovacancy undergo the Jahn-Teller distortion by breaking the three-fold symmetry, and form a pentagonal and an enneahedral ring of $\mathrm{C}$ atoms (Fig. 4a) ${ }^{[34,35]}$. The relaxed danglingbond sites constitute an isosceles triangle with two $\mathrm{C}$ atoms at the base forming a weak bond, while the largest magnetic moment is presented at the apex. The calculated magnetic moment for the mono-vacancy is $1.4 \mu_{\mathrm{B}}$ and the formation energy is $7.56 \mathrm{eV}$.

Similar to the interactions of other types of local magnetic moments in bulk materials, the bipartite character is important in magnetic interactions of vacancies in graphene ${ }^{[36,37]}$. Multiple mono-vacancies exhibit FM (/anti-FM) interaction if they are in the same 
(/different) sublattice of graphene, as seen in Fig. 4b. Clustering of multiple vacancies creates a void, and leaves atoms with unpaired electrons (due to dangling-bonds) along the circumference (Figs. 4c-d). Large vacancy-clusters can have many unpaired electrons. However, the net magnetic moment is generally small due to the anti-FM interaction between two sublattices of graphene. Unpaired electrons in the same sublattice of graphene energetically prefer the FM interaction, whereas those in different sublattices favor antiFM ordering. In the relaxed voids, instant reconstruction of bonds occurs for some atoms with dangling bonds. Hence, the difference between the numbers of dangling-bond sites in two sublattices is generally small, typically one to three. In the case of graphene nanoribbons, it has been predicted that the zigzag edge exhibits FM ordering as it contains atoms of only one sub-lattice type ${ }^{[38-41]}$. As shown in Fig. 4e, spin moments in GnPs are mainly distributed at the edge carbon atoms, and decay exponentially into the center of the ribbon.

(a)

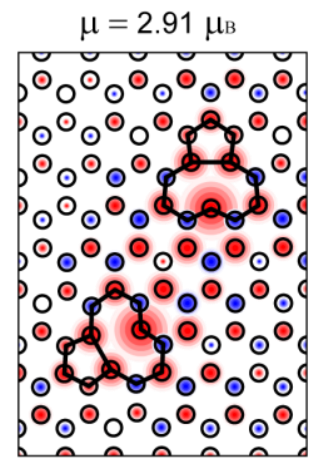

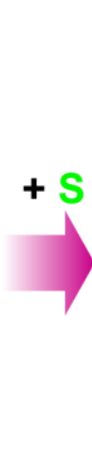

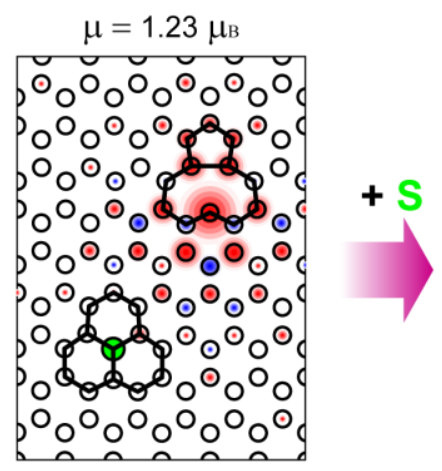

(c) $\quad \mu=0.00 \mu_{\mathrm{B}}$

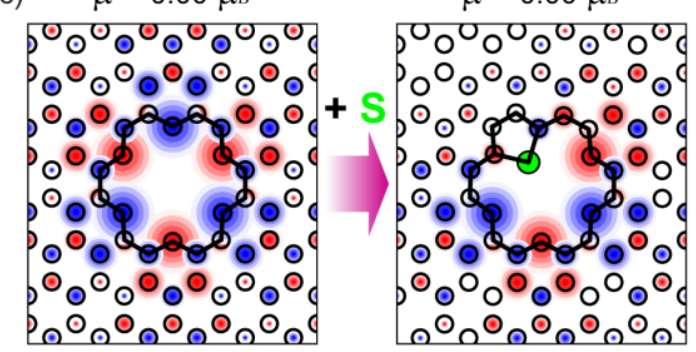

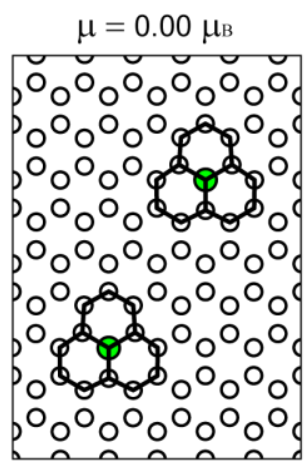

(b)

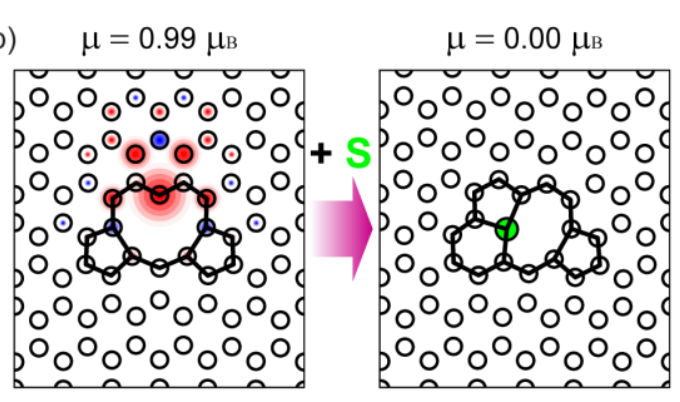

Figure 5. Demagnetization effect of sulfur in graphene with vacancies. In (a), two ferromagnetically interacting mono-vacancies are gradually demagnetized by successive additions of two sulfur atoms. Similarly, local magnetic moments of clusters of vacancies are quenched by attached sulfur atoms in (b) and (c).

Consistent with experimental results (cf. Fig 1), we find that S-doping suppresses magnetic moments induced by dangling-bonds at vacancy sites in graphene lattice. As shown in Fig. $5 \mathrm{a}$, the FM interaction between two mono-vacancies located within the interaction range $\left(<10 \mathrm{~nm}{ }^{[19,20]}\right)$, results in a net magnetic moment of $2.9 \mu_{\mathrm{B}}$. Doping with a single sulfur atom that occupies one of the two available vacant substitutional sites leads to a loss of magnetic moment to $1.2 \mu_{\mathrm{B}}$. Additional sulfur atom occupies the remaining vacant site and 
completely quenches the magnetism. Similar results are observed for graphene with trivacancy, shown in Fig. 5b, where a substitutional sulfur atom leads to a complete loss of magnetic moment from $1.0 \mu_{\mathrm{B}}$ to zero. Fig. $5 \mathrm{c}$ shows a special case where the distribution of local magnetic moments is induced by six dangling-bond sites in the cluster of six vacancies. Up and down spin densities are symmetrically distributed in two sublattices of graphene in the vicinity of the cluster, but they balance out each other and yield no net magnetic moment. The attached sulfur atom negates nearby local moment, but the net magnetic moment of the system remains zero. In all cases we investigated, we found that the attached sulfur atom itself does not impart any magnetism to any non-magnetic structures in graphene.
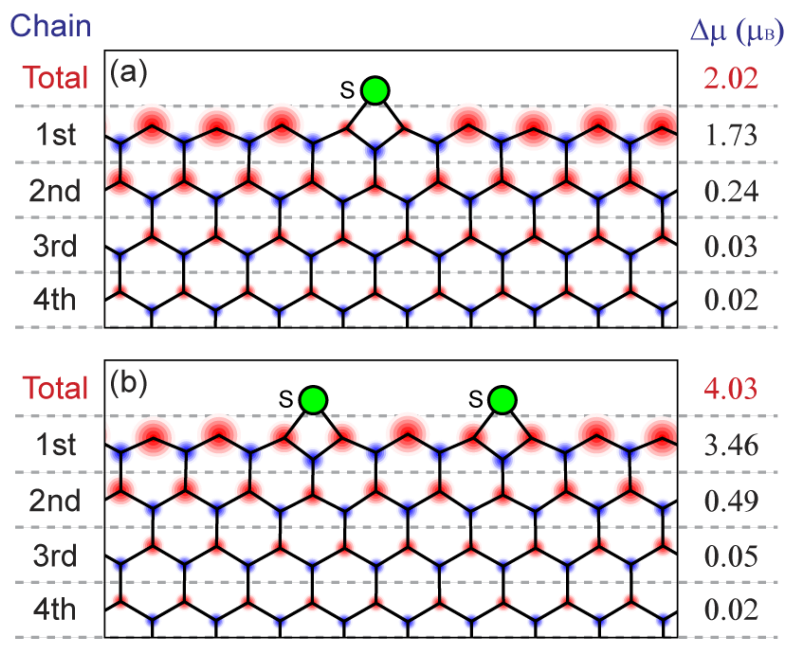

\section{1.}

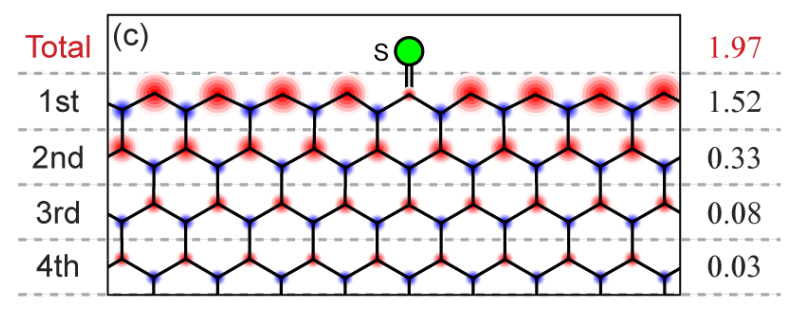

Figure 6. Demagnetization effect of sulfur in zigzag edges of graphene. Regardless of the type of bond, about $2 \mu_{B}$ of reduction per sulfur atom in the total magnetic moment of the zigzag edge is observed.

The magnetic properties of the zigzag graphene edges are also affected by the addition of sulfur atoms. As shown in Fig. 6, S-dopants locally demagnetize graphene along zigzag edges with approximately $\sim 2 \mu_{\mathrm{B}}$ reduction in magnetic moment per S-dopant in the net magnetic moment. When a S-dopant is present, it quenches the local magnetic moment by bonding unpaired electrons of edge carbon atom. Nonetheless, the FM ordering of spin moments along zigzag chains remains persistent even after the addition of multiple $\mathrm{S}$ atoms. 


\section{Conclusions}

In summary, our experiments showed that the magnetism in graphene is sensitive to the nature of the defects. While pristine graphene with naturally occurring edges and vacancies (i.e., intrinsic defects) exhibits a non-zero magnetic moment, the addition of S-dopants was found to quench this magnetic ordering. In fact, we found that sulfur doping drastically changes the magnetic behavior of the as-prepared samples. The zero-field-cooling (ZFC) and field-cooling (FC) in M vs. T measurements indicated the co-existence of large amount of super-paramagnetic domains along with antiferromagnetic domains in all the samples. From our DFT calculations, $\mathrm{S}$ dopants in GnPs were found to be attracted to defected sites such as vacancies, vacancy clusters, and edges. Indeed, S-dopants were observed to saturate dangling bonds and quench local magnetic moment of defect structures and ultimately result in a diamagnetic response, weakly ferromagnetic, and antiferromagnetic ordering concurring with our experimental findings. When taken together, our experimentally observed demagnetization in S-doped GnPs and theoretical calculations confirm that the net magnetization in pristine graphene arises from the presence of entropically necessary defects and not just metal impurities.

\section{Acknowledgements}

The computational work was supported by DOE-BES-DMS (DEFG02-99ER45795). We used computational resources of the NERSC, supported by the U.S. DOE (DE-AC0205CH11231), and the Ohio Supercomputing Center. J. H would like to acknowledge the support of NSF DMR 1307740. A. M. R and R. P acknowledge the support from US National Science Foundation grant CMMI-1246800 award. The authors acknowledge Drs. E. M. Wylie and Brian Powell for their help with ICP-MS.

\section{Author contribution}

J.Z., R.P. and A.M.R. designed the experiments. J.Z., and L.O. synthesized pristine and doped samples. J.Z., J.H., A.H. and A.Z. have done the magnetic characterization. P.A. performed XPS measurements. H.P., A.W. and J.W. performed the DFT calculations. J.Z., R.P., J.H. and A.M.R. analyzed the data. J.Z., R.P., A.M.R., H.P. and A.W. drafted the results.

\section{References}

[1] L.E. Hueso, J.M. Pruneda, V. Ferrari, G. Burnell, J. P. Valdes-Herrera, B. D. Simons, P. B. Littlewood, E. Artacho, A. Fert, and N. D. Mathur, Nature, 2007, 445(7126), 410-413. 
[2] K. Tsukagoshi, B.W. Alphenaar, and H. Ago, Nature, 1999, 401(6753), 572-574.

[3] J. W. McClure, Physical Review, 1956, 104(3), 666.

[4] P. Esquinazi, A. Setzer, R. Ho"hne, C. Semmelhack, Y. Kopelevich, D. Spemann, T. Butz, B. Kohlstrunk, and M. Lo"sche, Phys. Rev. B, 2002, 66, 024429.

[5] P. O. Lehtinen, A. S. Foster, Y. Ma, A. V. Krasheninnikov, and R. M. Nieminen, Phys. Rev. Lett., 2004, 93(18), 187202.

[6] O. V. Yazyev, Phys. Rev. Lett., 2008, 101(3), 037203.

[7] K. Sawada, F. Ishii, M. Saito, S. Okada, T. Kawai, Nano Lett., 2009, 9, 269.

[8] (a)K. Wakabayashi, M. Fujita, H. Ajiki and M. Sigrist, Phys. Rev. B, 2009, 59, 8271

(b) K. Kusakabe and M. Maruyama, Phys. Rev. B, 2003, 67, 092406.

[9] T. L. Makarova, B. Sundqvist, R. Höhne, P. Esquinazi, Y. Kopelevich, P. Scharff, V. A. Davydov, L. S. Kashevarova and A. V. Rakhmanina, Nature, 2001, 413, 716-718; see however Retraction: Nature 2006, 440, 707.

[10] A. V. Rode, E. G. Gamaly, A. G. Christy, JG Fitz Gerald, S. T. Hyde, R. G. Elliman, B. Luther-Davies, A. I. Veinger, J. Androulakis, and J. Giapintzakis, Phys. Rev. B, 2004, 70, 054407.

[11] H. Ohldag, T. Tyliszczak, R. Höhne, D. Spemann, P. Esquinazi, M. Ungureanu, and T. Butz, Phys. Rev. Lett., 2007, 98, 187204.

[12] A. K. Geim, and K. S. Novoselov, Nature materials, 2007. 6(3), 183-191.

[13] A. H. Castro Neto, F. Guinea, N. M. R. Peres, K. S. Novoselov and A. K. Geim, Rev. Mod. Phys., 2009, 81, 109.

[14] D. Soriano, F. Muñoz-Rojas, J. Fernández-Rossier and J. J. Palacios, Phys. Rev. $B, 2010,81,165409$.

[15] F. J. Culchac, A. Latgé and A. T. Costa, New J. Phys., 2011, 13, 033028.

[16] H. Feldner,; Z. Y. Meng,; T. C. Lang, F. F. Assaad, S. Wessel and A. Honecker, Phys. Rev. Lett., 2008, 106, 226401.

[17] Y. W. Son, M. L. Cohen and S. G. Louie, Nature, 2006, 444, 347.

[18] O. V. Yazyev, Rep. Prog. Phys. 2010, 73, 056501.

[19] M. Sepioni, R. R. Nair, S. Rablen, J. Narayanan, F. Tuna, R. Winpenny, A. K. Geim, and I.V. Grigorieva, Phys. Rev. Lett., 2010, 105, 207205.

[20] R. R. Nair, M. Sepioni, I-Ling Tsai, O. Lehtinen, J. Keinonen, A. V. Krasheninnikov, T. Thomson, A. K. Geim and I. V. Grigorieva, Nature Physics, 2012, 8, 199.

[21] Y. Wang, Y. Huang, Y. Song, X. Zhang, Y. Ma, J. Liang and Y. Chen, Nano Lett., 2009, 9, 220.

[22] H. S. S. Ramakrishna Matte, K. S. Subrahmanyam, and C. N. R. Rao, J. Phys. Chem. C, 2009, 113, 9983. 
[23] S. S. Rao, S. Narayana Jammalamadaka, A. Stesmans, V. V. Moshchalkov, J. van Tol, D. V. Kosynkin, A. Higginbotham-Duque and J. M. Tour, Nano Lett., 2012, 12, 1210.

[24] R. Podila, W. Queen, A. Nath, J. T. Arantes, A. L. Schoenhalz, A. Fazzio, G. M. Dalpian, J. He, S. J. Hwu, M. J. Skove and A. M. Rao, Nano Lett., 2010, 10 (4), 1383-1386.

[25] B. B. Straumal, A. A. Mazilkin, S. G. Protasova, A. A. Myatiev, P. B. Straumal, G. Schütz, P. A. van Aken, E. Goering and B. Baretzky, Phys. Rev. B, 2009, 79(20), 205206.

[26] T. Tietze, P. Audehm, Y. Chen, G. Schütz, B. B. Straumal, S. G. Protasova, A. A. Mazilkin, P. B. Straumal, T. Prokscha, H. Luetkens, Z. Salman, A. Suter, B. Baretzky, K. Fink, W. Wenzel, D. Danilov and E. Goering, Scientific reports, 2015, 5, 8871 .

[27] S. G. Protasova, B. B. Straumal, A. A. Mazilkin, S. V. Stakhanova, P. B. Straumal, and B. Baretzky, Journal of Materials Science, 2014, 49(13), 44904498.

[28] P. E. Blochl, Phys. Rev. B, 1994, 50, 17953.

[29] G. Kresse and D. Joubert, Phys. Rev. B, 1999, 59, 1758.

[30] G. Kresse and J. Furthmuller, Phys. Rev. B, 1996, 54, 11169.

[31] G. Kresse and J. Furthmuller, Computational Materials Science, 1996, 6, 15.

[32] J. P. Perdew, K. Burke, and M. Ernzerhof, Phys. Rev. Lett., 1996, 77, 3865.

[33] 25 E. Z. Kurmaev, A. V. Galakhov, A. Moewes, S. Moehlecke, \& Y. Kopelevich, Phys. Rev. B, 2002, 66, 193402.

[34] Y. C. Ma, P. O. Lehtinen, A. S. Foster, and R. M. Nieminen, New J. Phys., 2004, 6, 68.

[35] A. A. El-Barbary, R. H. Telling, C. P. Ewels, M. I. Heggie, and P. R. Briddon, Phys. Rev. B, 2003, 68, 144107.

[36] H. Park, A. Wadehra, J. W. Wilkins, and A. H. C. Neto, Phys. Rev. B, 2013, 87, 085441.

[37] H. Park, A. Wadehra, J. W. Wilkins, and A. H. C. Neto, Appl. Phys. Lett. 2012, 100, 253115.

[38] F. Wu, E. J. Kan, H. J. Xiang, S. H. Wei, M. H. Whangbo, and J. L. Yang, Appl. Phys. Lett., 2009, 94(22), 223105.

[39] Y. W. Son, M. L. Cohen, and S. G. Louie, Nature, 2006, 444, 347.

[40] L. Chen, L. Guo, Z. Li, H. Zhang, J. Lin, J. Huang, S. Jin, and X. Chen, Scientific reports, 2013, 3, 2599.

[41] S. Zhao, Y. Lü, W. Lü, W. Liang, and E. Wang, Chin. Phys. B, 2014, 23(6), 067305.

[42] B. C. Zhao, Y. Q. Ma, W. H. Song and Y. P. Sun, Phys. Lett. A, 2006, 354, 472476. 